\title{
Characteristics of P-glycoprotein (PgP) Upregulated in Chronic Cocaine Users and HIV Infected Persons
} Pablo Lopez, Rosa Velez, Vanessa Rivera, Nayra Rodriguez and Yasuhiro Yamamura*‡

Address: Ponce School of Medicine AIDS Research Program, Ponce, PR 00716-2348, USA

Email: Yasuhiro Yamamura* - yyamamura@psm.edu

* Corresponding author ‡Presenting author

from 2005 International Meeting of The Institute of Human Virology Baltimore, USA, 29 August - 2 September 2005

Published: 8 December 2005

Retrovirology 2005, 2(SuppI I):PI42 doi:I0.I I86/I742-4690-2-SI-PI42

\section{Background}

Chronic cocaine use and HIV independently upregulate cellular expression of a membrane bound efflux protein, Pgp, reducing the bioavailability of HIV-protease inhibitor drugs. Pgp possesses two functionally distinct binding sites that are associated with its efflux function. It is not known if Pgp upregulation by cocaine and/or HIV infection differently modifies the function of the two binding sites of Pgp.

\section{Materials and methods}

Peripheral blood was obtained from HIV negative chronic cocaine users. Peripheral blood mononuclear cells (PBMCs) were isolated and their Pgp and HIV coreceptor expression was assessed by Flow cytometry. Efflux function of Pgp was assessed also by flow cytometry by measuring the uptakes by Pgp positive CD4 T cells of two different substrates, Rhodamine 123 (R) and Hoechst $33342(\mathrm{H})$. Cyclosporine-A and Colchicines were used to induce R- and $\mathrm{H}$-site specific inhibition of the efflux functions.

\section{Results}

Even though both HIV infection and chronic cocaine use independently upregulated Pgp expression on CD4 T cells, each induced a different level of alteration of R- and $\mathrm{H}$-site specific efflux function.

\section{Conclusion}

HIV infection and chronic cocaine use similarly increased Pgp expression but presumably induced configurationally different alterations of the efflux pump molecule. 